\title{
Perioperative outcomes among chronic opioid users who receive lobectomy for non-small cell lung cancer
}

\author{
David B. Nelson, MD, MSc, ${ }^{a}$ Jiangong Niu, PhD, ${ }^{b}$ Kyle G. Mitchell, MD, ${ }^{a}$ Mara B. Antonoff, MD, ${ }^{a}$ \\ Sharon H. Giordano, MD, MPH, ${ }^{\text {b,c }}$ Wayne L. Hofstetter, MD, ${ }^{a}$ Ara A. Vaporciyan, MD, ${ }^{a}$ Boris Sepesi, MD, ${ }^{2}$ \\ Reza J. Mehran, MD, ${ }^{\mathrm{a}}$ and David C. Rice, $\mathrm{MB}, \mathrm{BCh}^{\mathrm{a}}$
}

\section{ABSTRACT}

Objective: We sought to identify whether chronic opioid users are at increased risk for complications or hospital readmission following lobectomy for nonsmall cell lung cancer.

Methods: The National Cancer Institute Surveillance, Epidemiology, and End Results-Medicare database was queried to identify patients older than age 65 years who received a lobectomy for non-small cell lung cancer. Chronic opioid users were identified through Medicare Part D records and were defined as those with $>120$ cumulative days of opioid supply for the year before surgery. A systematic 1:2 propensity matching was performed among chronic opioid users.

Results: Six thousand four hundred thirty-seven patients were identified, among whom 3627 (56\%) were opioid naïve, 1866 (29\%) were intermittent opioid users, and $944(15 \%)$ were chronic opioid users. After propensity matching, 30-day mortality and 90-day mortality were nearly 2-fold higher among chronic opioid users compared with nonchronic users. In addition, length of stay and hospital charges were increased among chronic opioid users (median, 6 vs 7 days and mean increase, $\$ 12,526$, respectively). Multivariable analysis revealed that intermittent opioid users and chronic opioid users were associated with an increased risk of 90-day hospital readmission compared with opioid-naive patients (odds ratio, $1.35 ; 95 \%$ confidence interval, $1.07-1.71$ and odds ratio, $1.72 ; 95 \%$ confidence interval, 1.40-2.12, respectively), predominantly burdened by infectious, renal, and pulmonary causes.

Conclusions: Patients who chronically use opioids before lobectomy represent high-risk patients. The risk of 30- and 90-day mortality, length of stay, hospital charges, and 90-day readmission after lobectomy among chronic opioid users are substantially elevated. (J Thorac Cardiovasc Surg 2020;159:691-702)

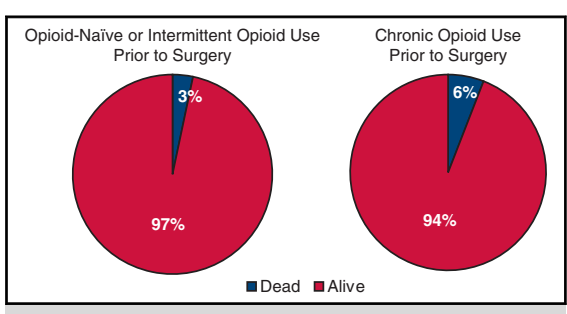

Propensity matched 30-day mortality among chronic users of opioid drugs.

Central Message

Patients who chronically use opioids before lobectomy represent high-risk surgical patients.

\section{Perspective}

In a propensity score-matched analysis, we found that chronic opioid use before lobectomy was associated with a nearly doubled 30 - and 90-day risk of mortality. In addition, chronic opioid use was associated with prolonged length of stay, increased hospital charges, and increased 90-day hospital readmission. Screening to identify chronic opioid use before elective lobectomy should be performed.

See Commentary on page 703 .

\footnotetext{
From the Departments of ${ }^{\text {a }}$ Thoracic and Cardiovascular Surgery, ${ }^{b}$ Health Services Research, and ${ }^{\mathrm{c}}$ Breast Medical Oncology, The University of Texas MD Anderson Cancer Center, Houston, Tex.

Supported in part by the National Cancer Institute (grant No. P30 CA016672) and by departmental support. Dr Giordano is supported by the Cancer Prevention and Research Institute of Texas (grant No. RP160674).

Drs Mehran and Rice contributed equally to this article.

Read at the 99th Annual Meeting of The American Association for Thoracic Surgery, Toronto, Ontario, Canada, May 4-7, 2019.

Received for publication May 4, 2019; revisions received Aug 21, 2019; accepted for publication Sept 4, 2019.

Address for reprints: David C. Rice, MB, BCh, Department of Thoracic and Cardiovascular Surgery, The University of Texas MD Anderson Cancer Center, 1400 Pressler St, Unit 1489, Houston, TX 77030 (E-mail: drice@mdanderson.org). $0022-5223 / \$ 36.00$

Copyright (C) 2019 by The American Association for Thoracic Surgery https://doi.org/10.1016/j.jtcvs.2019.09.059
}

Opioid use has drastically risen during the past 2 decades, with hydrocodone use increasing by more than 2 -fold and oxycodone use increasing by 5 -fold. ${ }^{1}$ Although the prevalence of opioid addiction has increased substantially, the influence of chronic opioid use before surgery on perioperative outcomes of patients undergoing general

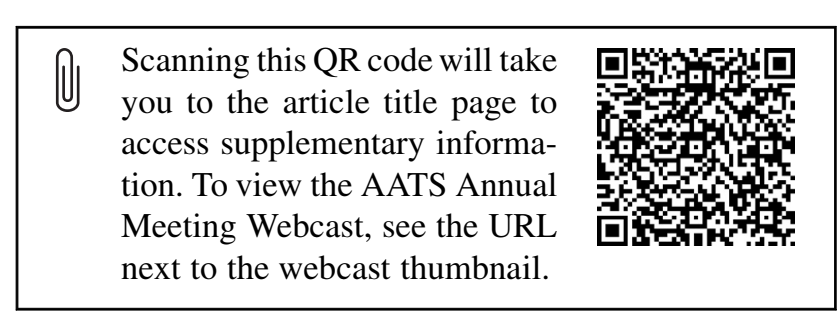




\section{Abbreviations and Acronyms \\ NSCLC $=$ non-small cell lung cancer \\ OME $=$ oral morphine equivalent \\ SEER = Surveillance, Epidemiology, and End Results}

thoracic surgery remains to be well described. Several retrospective studies have highlighted increased costs and morbidity encountered among opioid users who undergo surgery. $^{2-4}$ Although these results may represent other factors intrinsic to those who chronically use opioids, they nonetheless highlight increased perioperative burden among this population. Furthermore, opioid use may promote immunosuppression, ${ }^{5}$ raise the risk of sepsis in animal models, ${ }^{6}$ and are also implicated in impaired wound healing. ${ }^{7}$ The purpose of this study was to characterize early perioperative outcomes, including morbidity, mortality, and readmission burden among chronic opioid users who undergo lobectomy for non-small cell lung cancer (NSCLC). We hypothesized that chronic opioid users demonstrate worse perioperative outcomes compared with intermittent or naïve opioid users.

\section{METHODS}

\section{Database Information}

A query of the National Cancer Institute Division of Cancer Control and Population Sciences Surveillance, Epidemiology and End Results (SEER)-Medicare linked database (https://healthcaredelivery.cancer. gov/seermedicare/) was performed. Medicare eligibility includes $97 \%$ of those who are aged 65 years or older with claims data available for linkage with those who have fee-for-service coverage. The SEER database is supported by the National Cancer Institute and maintains cancer incidence and survival data from population-based cancer registries. ${ }^{8}$ Approximately $35 \%$ of the US population is covered by the SEER database. The SEER-Medicare linkage led to $93 \%$ of persons who are aged 65 years and older and registered within the SEER database to be matched to the Medicare database. Part D coverage, which provides additional coverage for prescription medications, is available for approximately $60 \%$ of Medicare beneficiaries. This study was approved by the MD Anderson Cancer Center Institutional Review Board with a waiver of informed consent.

\section{Patient Selection Criteria}

Patients who were older than age 65 years and who received a lobectomy for NSCLC between 2008 to 2013 were selected for analysis. To ensure complete claims data, including prescription records, enrollment in Medicare parts A, B, and D for 12 months before surgery without enrollment in an health maintenance organization was required. Patients were required to have histologic confirmation of diagnosis and to undergo surgery within 6 months of diagnosis or be diagnosed as late as 30 days after surgery. Patients who were diagnosed at autopsy or death certificate were excluded.

\section{Variable Definitions}

Opioid use was identified by Part D records according to the name of opioid drug prescribed, dose, and quantity filled. Patients were categorized as chronic opioid users if at least 120 cumulative days of supply were filled 1 year before surgery. Opioid-naïve patients were those with no filled opioid prescription for the year before surgery, and intermittent opioid users filled $<120$ cumulative days of supply. We excluded opioid prescriptions filled within 30 days of surgery because some surgeons will provide patients with an opioid prescription intended to treat postoperative pain in preparation for surgery. These definitions reflect those previously used.

NSCLC histology was identified using International Classification of Diseases for Oncology, 3rd Edition, histology codes (Appendix E1 and Table E1). Charlson comorbidity scores were calculated using Medicare Provider Analysis and Review, National Claims History, and outpatient claims for 1 year before surgery as described using the Klabunde algorithm. ${ }^{10,11}$ Adjuvant chemotherapy and radiation therapy was identified using Medicare claims. Stage was defined according to the American Joint Committee on Cancer Collaborative Staging Manual, sixth edition, collaborative stage. Education level, poverty level, and residence in an urban or rural environment were identified through use of census data corresponding to the ZIP code of residence of the patient. To protect the confidentiality of Medicare beneficiaries and according to SEER-Medicare database guidelines, cells with counts $<11$ were suppressed.

\section{Statistical Analysis}

Comparisons between cohorts among categorical variables were performed using either $\chi^{2}$ test or Fischer exact test. Length of stay was compared using Kruskal-Wallis test. Charges were compared using a generalized linear model with a log-link and gamma distribution. Monthly oral morphine equivalent (OME) was calculated as the quantity of the opioid filled multiplied by the OME of each opioid drug. Monthly OME was reported as the mean of the cohort. Monthly OME use was compared using the Wilcoxon signed-rank test. A propensity score model predicting the likelihood of chronic opioid use before surgery was constructed using an a priori selected multivariable logistic regression model. Variables included in the propensity score model were age, race/ethnicity, sex, year of diagnosis, urban/rural environment, marital status, poverty level, education level, Charlson comorbidity score, tumor histology, tumor size, tumor grade, stage, surgical approach (video-assisted thoracoscopic surgery vs open), and receipt of any neoadjuvant therapy. After propensity score matching, cohorts were well balanced with standardized mean differences $<10 \%$ (Figures E1 and E2). In addition, there were no significant differences between cohorts (Table E2). Statistical tests were 2-sided. All statistical analysis was performed with use of SAS version 9.3 (SAS Institute Inc, Cary, NC).

\section{RESULTS}

\section{Characteristics of Chronic Opioid Users}

Six thousand four hundred thirty-seven patients were identified as having undergone a lobectomy for NSCLC between 2008-2013 (Figure 1). Among these, 3627 patients $(56 \%)$ were opioid-naive for the year before surgery. One thousand eight hundred sixty-six patients $(29 \%)$ were intermittent opioid users, and 944 patients $(15 \%)$ were chronic opioid users (Table 1). Chronic opioid use was defined as filling $>120$ cumulative days' worth of opioid drugs during the year before surgery. Chronic opioid use before surgery was independently associated with younger age, female sex, unmarried status, worse comorbidities, residence in a ZIP code with higher poverty level, race/ethnicity, and squamous cell histology (Table 2). 


\section{Operative Morbidity and Mortality Is Higher Among Chronic Opioid Users Who Undergo Lobectomy}

Complications, length of stay, and hospital charges were significantly higher among chronic opioid users compared with either naive or intermittent opioid users (Table 3). In addition, 30-day and 90-day mortality were significantly higher among chronic opioid drug users.
The 90-day mortality was 5.5\% among opioid-naïve patients, 6.4\% among intermittent opioid users, and $9.4 \%$ among chronic opioid users $(P=.001)$. The 90 -day hospital readmission rate was $20 \%$ among opioid-naïve patients, $24 \%$ among intermittent opioid users, and $31 \%$ among chronic opioid users $(P<.001)$. Chronic opioid users demonstrated an increased readmission burden

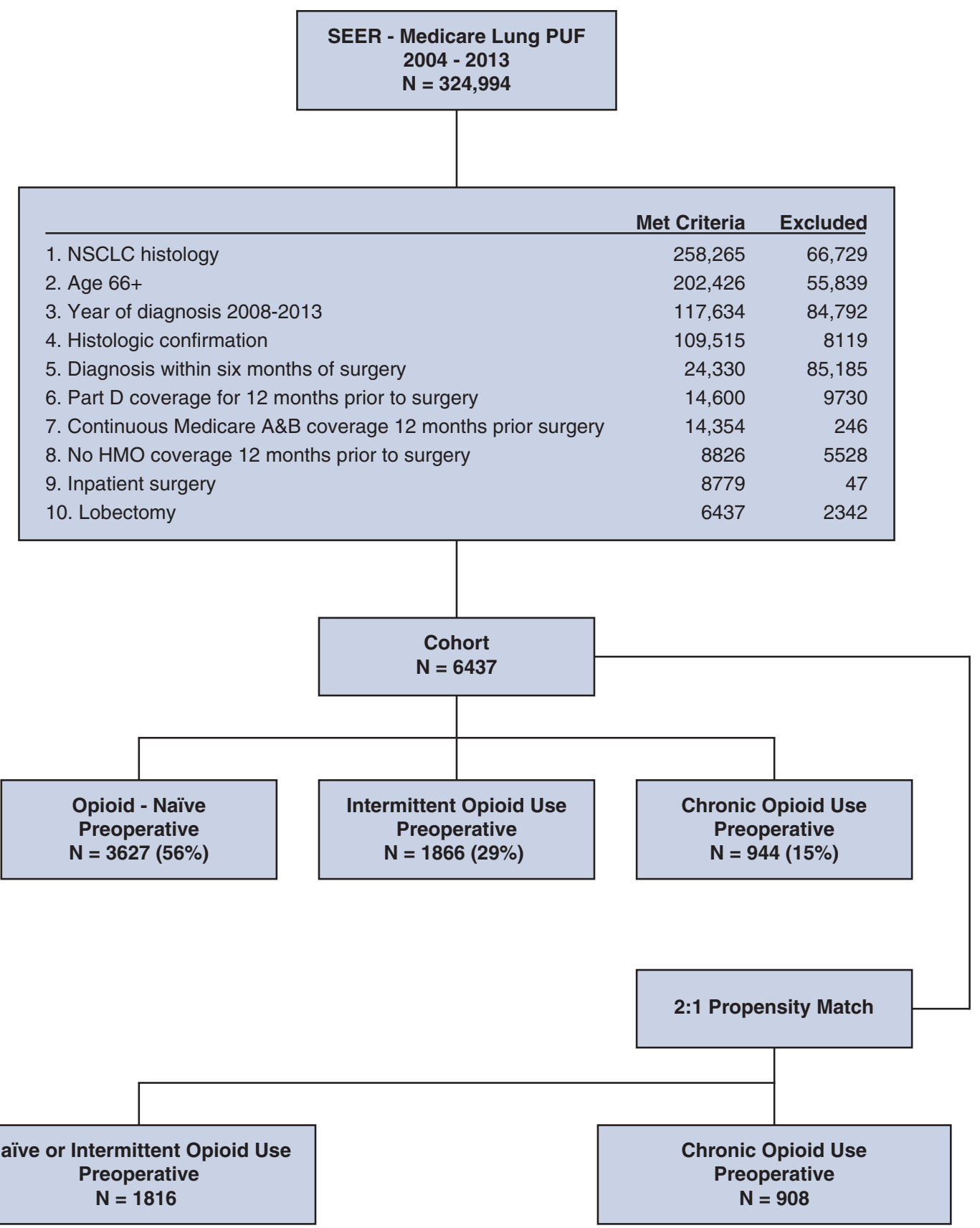

FIGURE 1. Study flowchart indication patient selection criteria. SEER, Surveillance, Epidemiology, and End Results; PUF, Participant User Files; NSCLC, non-small cell lung cancer; $H M O$, health maintenance organization. 
TABLE 1. Unmatched patient, tumor, and treatment characteristics

\begin{tabular}{|c|c|c|c|c|}
\hline \multirow[b]{2}{*}{ Characteristic } & \multicolumn{2}{|c|}{ No preoperative chronic opioid use $(n=5493)$} & \multirow[b]{2}{*}{$\begin{array}{l}\text { Preoperative chronic } \\
\text { opioid use }(n=944)\end{array}$} & \multirow[b]{2}{*}{$P$ value } \\
\hline & $\begin{array}{c}\text { Opioid naïve } \\
(\mathrm{n}=3627)\end{array}$ & $\begin{array}{c}\text { Intermittent } \\
\text { opioid use }(n=1866)\end{array}$ & & \\
\hline Age $(y)$ & & & & $<.001$ \\
\hline $66-70$ & $1067(29)$ & $653(35)$ & $384(41)$ & \\
\hline $71-75$ & $1092(30)$ & $570(31)$ & $295(31)$ & \\
\hline $76-80$ & $880(24)$ & $406(22)$ & $186(20)$ & \\
\hline$>80$ & $588(16)$ & $237(13)$ & $79(8)$ & \\
\hline Sex & & & & .002 \\
\hline Male & $1588(44)$ & $765(41)$ & $355(38)$ & \\
\hline Female & $2039(56)$ & $1101(59)$ & $589(62)$ & \\
\hline Year of diagnosis & & & & .601 \\
\hline 2008 & $633(17)$ & $306(16)$ & $162(17)$ & \\
\hline 2009 & $596(16)$ & $324(17)$ & $145(15)$ & \\
\hline 2010 & 607 (17) & $314(17)$ & $153(16)$ & \\
\hline 2011 & $570(16)$ & $299(16)$ & $165(17)$ & \\
\hline 2012 & $574(16)$ & 315 (17) & $168(18)$ & \\
\hline 2013 & $647(18)$ & 308 (17) & $151(14)$ & \\
\hline Race/ethnicity & & & & $<.001$ \\
\hline Non-Hispanic white & $2968(82)$ & $1593(85)$ & $800(85)$ & \\
\hline Non-Hispanic black & $180(5)$ & $99(5)$ & $76(8)$ & \\
\hline Hispanic & $171(5)$ & $87(5)$ & $55(6)$ & \\
\hline Non-Hispanic other & $308(8)$ & $87(5)$ & $13(1)$ & \\
\hline Urban/rural & & & & $<.001$ \\
\hline Big metro & $2093(58)$ & $960(51)$ & $444(47)$ & \\
\hline Less urban & $323(9)$ & $219(12)$ & $120(13)$ & \\
\hline Metro & $943(26)$ & $512(27)$ & $275(16)$ & \\
\hline Rural/unknown & $88(2)$ & $44(2)$ & $41(4)$ & \\
\hline Urban & $180(5)$ & $131(7)$ & $64(7)$ & \\
\hline Marital status & & & & $<.001$ \\
\hline Married & $1927(53)$ & $976(52)$ & 407 (43) & \\
\hline Not married & $1567(43)$ & 807 (43) & $509(54)$ & \\
\hline Unknown & $133(4)$ & $83(4)$ & $28(3)$ & \\
\hline$\%$ Below poverty line within ZIP code & & & & $<.001$ \\
\hline $0-5.4$ & $1001(28)$ & $466(25)$ & $136(14)$ & \\
\hline $5.4-10.3$ & $843(23)$ & $383(26)$ & $276(29)$ & \\
\hline $10.3-18.8$ & $970(27)$ & $455(24)$ & $176(19)$ & \\
\hline$\geq 18.8$ & $813(50)$ & $462(25)$ & $356(38)$ & \\
\hline$\%$ With no high school education within ZIP code & & & & $<.001$ \\
\hline $0-5.8$ & $1013(28)$ & $452(24)$ & $140(15)$ & \\
\hline $5.8-11.0$ & $872(24)$ & $443(24)$ & $284(30)$ & \\
\hline $11.0-20.0$ & $926(26)$ & $481(26)$ & $195(21)$ & \\
\hline$\geq 20.0$ & $816(23)$ & $490(26)$ & $325(34)$ & \\
\hline Charlson comorbidity score & & & & $<.001$ \\
\hline 0 & $1697(47)$ & $722(39)$ & $252(27)$ & \\
\hline 1 & $1168(57)$ & $603(32)$ & $294(31)$ & \\
\hline $2+$ & $762(21)$ & $541(29)$ & $398(42)$ & \\
\hline Histology & & & & $<.001$ \\
\hline Adenocarcinoma & $2310(64)$ & $1148(62)$ & $521(55)$ & \\
\hline Squamous & $981(27)$ & $539(29)$ & $337(36)$ & \\
\hline Large cell & $80(2)$ & $37(2)$ & $34(4)$ & \\
\hline Other/NOS & $256(7)$ & $142(8)$ & $52(6)$ & \\
\hline
\end{tabular}


TABLE 1. Continued

\begin{tabular}{|c|c|c|c|c|}
\hline \multirow[b]{2}{*}{ Characteristic } & \multicolumn{2}{|c|}{ No preoperative chronic opioid use $(n=5493)$} & \multirow[b]{2}{*}{$\begin{array}{l}\text { Preoperative chronic } \\
\text { opioid use }(n=944)\end{array}$} & \multirow[b]{2}{*}{$P$ value } \\
\hline & $\begin{array}{c}\text { Opioid naïve } \\
(n=3627)\end{array}$ & $\begin{array}{c}\text { Intermittent } \\
\text { opioid use }(n=1866)\end{array}$ & & \\
\hline Grade & & & & .037 \\
\hline Well & $591(16)$ & $295(16)$ & $123(13)$ & \\
\hline Moderate & $1553(43)$ & $749(40)$ & $409(43)$ & \\
\hline Poor & $1220(34)$ & $664(36)$ & $347(37)$ & \\
\hline Unknown & $263(7)$ & $158(8)$ & $65(7)$ & \\
\hline Stage & & & & .647 \\
\hline I & $2383(66)$ & $1184(63)$ & $603(64)$ & \\
\hline II & $452(12)$ & $224(12)$ & $121(13)$ & \\
\hline III & $509(14)$ & $295(16)$ & $148(16)$ & \\
\hline IV & $133(4)$ & $83(4)$ & $39(4)$ & \\
\hline Unknown/0 & $145(4)$ & $80(31)$ & $33(3)$ & \\
\hline Tumor size $(\mathrm{cm})$ & & & & .083 \\
\hline$<2$ & $925(26)$ & $517(28)$ & $248(26)$ & \\
\hline $2-3$ & $1011(28)$ & $512(27)$ & $261(27)$ & \\
\hline $3-5$ & $1080(30)$ & $495(27)$ & $289(27)$ & \\
\hline$\geq 5$ & $611(17)$ & $342(18)$ & $146(15)$ & \\
\hline Approach & & & & $<.001$ \\
\hline Open & $2036(56)$ & $1123(60)$ & $609(65)$ & \\
\hline Video-assisted thoracoscopic surgery & $1591(44)$ & $743(40)$ & $335(35)$ & \\
\hline Chemotherapy & & & & $<.001$ \\
\hline None & $2595(72)$ & $1314(70)$ & $660(70)$ & \\
\hline Neoadjuvant & $143(4)$ & $145(8)$ & $71(8)$ & \\
\hline Adjuvant & $889(25)$ & $407(22)$ & $213(23)$ & \\
\hline Radiation & & & & $<.001$ \\
\hline None & $3097(85)$ & $1549(83)$ & $804(85)$ & \\
\hline Neoadjuvant & $54(2)$ & $81(4)$ & $26(3)$ & \\
\hline Adjuvant & $476(13)$ & $236(13)$ & $114(12)$ & \\
\hline
\end{tabular}

Values are presented as $\mathrm{n}(\%)$. NOS, Not otherwise specified.

related to infectious, renal, and pulmonary causes, with no relationship identified between preoperative opioid use and cardiac, hematologic, neurologic, or gastrointestinal reasons for readmission.

\section{Propensity-Matched Operative Outcomes Among Chronic Opioid Users}

Chronic opioid use remained associated with a nearly 2fold increase in 30-day and 90-day mortality after propensity matching (Table 4). There were no longer differences in the complication rate or 30-day readmission rate $(P=.079$ and $P=.301$, respectively). However, length of stay and hospital charges were significantly higher among chronic opioid users as opposed to opioid-naïve or intermittent opioid users ( $P=.002$ and $P<.001$, respectively). Readmissions relating to infectious, renal, pulmonary, and cardiac causes were also significantly higher among chronic users of opioid drugs $(P<.05$ for all $)$, with no significant differences in hospital readmissions relating to hematologic, neurologic, or gastrointestinal causes.

\section{Factors Associated With 90-Day Readmission Among Matched Cohort}

Compared with the opioid-naïve patients, intermediate and chronic opioid use before surgery were both associated with significant increases in 90-day hospital readmission (intermittent: odds ratio [OR], 1.35; 95\% confidence interval [CI], 1.07-1.71, and chronic: OR, $1.72 ; 95 \% \mathrm{CI}, 1.40-2.12)$. In addition, compared with those who chronically use opioids before surgery, intermittent opioid use was associated with a significant reduction in 90-day hospital readmission (OR, 0.79; $95 \%$ CI, 0.62-0.99). Other factors that were associated with 90-day hospital readmission included sex, marital status, residence in a ZIP code with a lower high school graduation rate, tumor grade, Charlson comorbidity score, and open approach (Table 5). However, age, year of diagnosis, race/ethnicity, American Joint Committee on Cancer stage, tumor histology, and receiving neoadjuvant therapy were not associated with 90-day hospital readmission. 
TABLE 2. Factors associated with chronic opioid use before surgery

\begin{tabular}{|c|c|c|}
\hline Factor & $\begin{array}{c}\text { Multivariable } \\
\text { Odds ratio }(95 \% \\
\text { Confidence } \\
\text { interval) }\end{array}$ & $P$ value \\
\hline Age group (y) & & $<.001$ \\
\hline$>80$ & Reference & \\
\hline $66-70$ & $2.35(1.80-3.06)$ & \\
\hline $71-75$ & $1.82(1.39-2.39)$ & \\
\hline $76-80$ & $1.53(1.15-2.04)$ & \\
\hline Sex & & $<.001$ \\
\hline Male & Reference & \\
\hline Female & $1.33(1.14-1.56)$ & \\
\hline Race/ethnicity & & $<.001$ \\
\hline Non-Hispanic white & Reference & \\
\hline Hispanic & $0.99(0.72-1.36)$ & \\
\hline Non-Hispanic black & $0.97(0.72-1.29)$ & \\
\hline Non-Hispanic other & $0.19(0.11-0.34)$ & \\
\hline Year of diagnosis & & .433 \\
\hline 2008 & Reference & \\
\hline 2009 & $0.88(0.68-1.13)$ & \\
\hline 2010 & $0.97(0.76-1.25)$ & \\
\hline 2011 & $1.08(0.85-1.39)$ & \\
\hline 2012 & $1.13(0.88-1.44)$ & \\
\hline 2013 & $0.95(0.74-1.22)$ & \\
\hline Urban/rural & & .825 \\
\hline Big metro & Reference & \\
\hline Metro & $1.04(0.87-1.24)$ & \\
\hline Urban & $1.02(0.75-1.39)$ & \\
\hline Less urban & $0.92(0.72-1.19)$ & \\
\hline Rural & $1.16(0.78-1.73)$ & \\
\hline Marriage status & & .002 \\
\hline Married & Reference & \\
\hline Not married & $1.29(1.10-1.51)$ & \\
\hline Unknown & $0.84(0.55-1.28)$ & \\
\hline $\begin{array}{l}\% \text { with no high school education } \\
\text { within ZIP code }\end{array}$ & & .138 \\
\hline $0-5.66$ & Reference & \\
\hline $5.66-10.5$ & $1.12(0.88-1.43)$ & \\
\hline $10.5-19.35$ & $1.35(1.04-1.74)$ & \\
\hline$\geq 19.35$ & $1.26(0.94-1.68)$ & \\
\hline $\begin{array}{l}\% \text { Below poverty line within ZIP } \\
\text { code }\end{array}$ & & $<.001$ \\
\hline $0-5.24$ & Reference & \\
\hline $5.24-10.03$ & $1.17(0.91-1.50)$ & \\
\hline $10.03-18.19$ & $1.81(1.39-2.35)$ & \\
\hline$\geq 18.19$ & $2.18(1.63-2.91)$ & \\
\hline Stage & & .898 \\
\hline I & Reference & \\
\hline 0 & $0.69(0.08-5.87)$ & \\
\hline II & $1.12(0.90-1.41)$ & \\
\hline III & $1.10(0.89-1.36)$ & \\
\hline IV & $1.09(0.75-1.59)$ & \\
\hline Unknown & $1.02(0.65-1.59)$ & \\
\hline
\end{tabular}

TABLE 2. Continued

\begin{tabular}{|c|c|c|}
\hline Factor & $\begin{array}{c}\text { Multivariable } \\
\text { Odds ratio }(95 \% \\
\text { Confidence } \\
\text { interval) }\end{array}$ & $P$ value \\
\hline Histology & & .043 \\
\hline Adenocarcinoma & Reference & \\
\hline Large cell & $1.50(0.98-2.29)$ & \\
\hline Other/NOS & $0.85(0.60-1.20)$ & \\
\hline Squamous & $1.18(1.00-1.40)$ & \\
\hline Tumor size $(\mathrm{cm})$ & & .371 \\
\hline$<2$ & Reference & \\
\hline $2-3$ & $0.96(0.79-1.17)$ & \\
\hline $3-5$ & $1.04(0.86-1.27)$ & \\
\hline$\geq 5$ & $0.85(0.67-1.09)$ & \\
\hline Approach & & .089 \\
\hline Open & Reference & \\
\hline $\begin{array}{l}\text { Video-assisted thoracoscopic } \\
\text { surgery }\end{array}$ & $0.87(0.74-1.02)$ & \\
\hline Neoadjuvant therapy & & .065 \\
\hline No & Reference & \\
\hline Yes & $1.31(0.98-1.74)$ & \\
\hline Grade & & .895 \\
\hline Well differentiated & Reference & \\
\hline Moderately differentiated & $1.07(0.84-1.35)$ & \\
\hline Poorly differentiated & $1.02(0.80-1.31)$ & \\
\hline Unknown & $0.97(0.68-1.37)$ & \\
\hline Charlson comorbidity score & & $<.001$ \\
\hline 0 & Reference & \\
\hline 1 & $1.51(1.26-1.81)$ & \\
\hline $2+$ & $2.80(2.34-3.34)$ & \\
\hline
\end{tabular}

NOS, Not otherwise specified.

\section{DISCUSSION}

We identified that chronic opioid use before lobectomy was associated with significantly increased hospital charges, length of stay, 30- and 90-day mortality, and hospital readmissions. To our knowledge, this represents the first large population-based study to investigate the influence of opioid use before lung surgery.

A propensity-matched study of 5.7 million adults who underwent cardiac surgery showed that an opioid use disorder before cardiac surgery was associated with a higher incidence of major complications, including blood transfusions, pulmonary embolism, prolonged mechanical ventilation, and pain. ${ }^{2}$ Similarly, worse perioperative outcomes among those who abuse or are dependent on opioid drugs have been reported among surgeries at several other sites, ${ }^{12-18}$ with increased costs, longer length of stay, increased morbidity, mortality, and 30-day readmission having all been reported. Complications or reason for readmission among these studies were predominantly infectious, pulmonary, and pain-related. ${ }^{12,13,18}$ In other studies, any amount of preoperative opioid use has also been 
TABLE 3. Unmatched early operative outcomes according to preoperative opioid use

\begin{tabular}{|c|c|c|c|c|}
\hline \multirow[b]{2}{*}{ Outcome } & \multicolumn{2}{|c|}{ No preoperative chronic opioid use $(n=5493)$} & \multirow[b]{2}{*}{$\begin{array}{c}\text { Preoperative chronic opioid } \\
\text { use }(n=944)\end{array}$} & \multirow[b]{2}{*}{$P$ value } \\
\hline & Opioid naïve $(\mathrm{N}=3627)$ & $\begin{array}{c}\text { Intermittent opioid use } \\
(\mathrm{n}=1866)\end{array}$ & & \\
\hline Complications & $2037(56)$ & $1116(59)$ & $610(64)$ & $<.001$ \\
\hline Length of hospital stay (d) & $6(4-9)$ & $6(4-9)$ & $7(5-10)$ & $<.001$ \\
\hline Discharge status & & & & $<.001$ \\
\hline Home & $3004(83)$ & $1521(82)$ & $689(73)$ & \\
\hline Skilled nursing facility & $372(10)$ & 215 (12) & $140(15)$ & \\
\hline Long-term care hospital & $54(1)$ & $23(1)$ & $29(3)$ & \\
\hline Rehabilitation facility & $82(2)$ & $39(2)$ & $26(3)$ & \\
\hline Not applicable/expired & $74(2)$ & $44(2)$ & $36(4)$ & \\
\hline Other & $41(1)$ & $24(1)$ & $24(3)$ & \\
\hline Mean hospital charges $(\$)$ & 104,781 & 107,375 & 119,253 & $<.001$ \\
\hline 30-d mortality & $127(3.5)$ & $72(3.9)$ & $58(6.1)$ & .001 \\
\hline 90-d mortality & $201(5.5)$ & $120(6.4)$ & $89(9.4)$ & $<.001$ \\
\hline 30-d hospital readmission & $427(12)$ & $262(14)$ & $152(16)$ & $<.001$ \\
\hline 90-d hospital readmission & $741(20)$ & $448(24)$ & $290(31)$ & $<.001$ \\
\hline \multicolumn{5}{|c|}{ 90-d hospital readmission rate according to readmission diagnosis* } \\
\hline Pulmonary & $371(10)$ & $233(12)$ & $147(16)$ & $<.001$ \\
\hline Cardiac & $260(7)$ & $140(8)$ & $85(9)$ & .163 \\
\hline Hematologic & $94(3)$ & $56(3)$ & $36(4)$ & .129 \\
\hline Renal & $284(8)$ & $155(8)$ & $114(12)$ & $<.001$ \\
\hline Infectious & $320(9)$ & $206(11)$ & $143(15)$ & $<.001$ \\
\hline Neurologic & $46(1)$ & $27(1)$ & $17(2)$ & .452 \\
\hline Gastrointestinal & $24(<1)$ & $15(<1)$ & $<11(<1)$ & 612 \\
\hline Pain & $11(<1)$ & $<11^{\dagger}$ & $<11 \dagger$ & .158 \\
\hline
\end{tabular}

Values are presented as $\mathrm{n}(\%)$ or median (interquartile range). $*$ More than one category may be present per patient; 287 patients were uncategorized. $\dagger$ Cells representing $\leq 10$ were suppressed per Surveillance, Epidemiology and End Results-Medicare guidelines.

independently associated with increased operative morbidity, length of stay, 30-day readmissions, and costs. $3,4,19,20$

Although methods to adjust for confounding were used for the above studies, patients who use opioid drugs before surgery harbor high-risk characteristics, as shown in our study and in other articles. Those who use opioid drugs before surgery are younger, are more likely to be a smoker or use illicit drugs, have worse pain, higher rates of depression, worse fibromyalgia scores, lower life satisfaction, and more medical comorbidities. ${ }^{21}$ Separately, younger age, race, female sex, and body mass index have been shown to be associated with chronic preoperative opioid use. ${ }^{22}$ In addition, opioid use has also been shown to be associated with poor compliance with treatment recommendations. ${ }^{23}$

Opioid drugs may plausibly increase morbidity through their immunosuppressive properties. ${ }^{5}$ In a mouse sepsis model with Streptococcus pneumoniae inoculation, chronic morphine treatment resulted in suppression of nuclear factor kappa light chain enhancer of activated B cells as well as modulation of macrophage inflammatory protein 2 and inflammatory cytokines. ${ }^{6}$ Delayed neutrophil recruitment, increased bacterial burden in the lung, spleen, and blood, and increased mortality was observed. Among observational studies, opioid drugs have been shown to be associated with an increased risk of community-acquired pneumonia $^{24}$ and also invasive pneumococcal disease. ${ }^{25}$ In addition, peripheral opioid receptors regulate wound healing through multiple levels, including modulating keratinocyte differentiation, migration, and adhesion. ${ }^{26,27}$ Mice treated with chronic morphine demonstrated delayed neutrophil and macrophage recruitment to wound sites, downregulation of neurokinin-1 neurokinin-2, decreased wound closure, and increased bacterial sepsis. ${ }^{28-30}$ Morphine decreases expression of hypoxia inducible factor 1-alpha and vascular endothelial growth factor, suppresses angiogenesis, and resulted in decreased wound closure in a rat model. ${ }^{31,32}$ Lastly, topical naloxone given to rats with type 1 diabetes show hastened wound closure with stimulation of angiogenesis. ${ }^{33}$ Perhaps not surprising based on these findings, postoperative opioid use remained an independent predictor of wound dehiscence after 
TABLE 4. Matched early operative outcomes according to preoperative opioid use

\begin{tabular}{|c|c|c|c|}
\hline Outcome & $\begin{array}{c}\text { Naïve or } \\
\text { intermittent } \\
\text { preoperative } \\
\text { opioid use } \\
(n=1816)\end{array}$ & $\begin{array}{c}\text { Chronic } \\
\text { preoperative } \\
\text { opioid use } \\
(\mathbf{n}=908)\end{array}$ & $P$ value \\
\hline Complications & $1103(61)$ & $583(64)$ & .079 \\
\hline Length of hospital stay (d) & $6(4-9)$ & $7(5-10)$ & .002 \\
\hline $\begin{array}{l}\text { Discharge status } \\
\text { Home } \\
\text { Skilled nursing facility } \\
\text { Long-term care hospital } \\
\text { Rehabilitation facility } \\
\text { Not applicable/expired } \\
\text { Other }\end{array}$ & $\begin{array}{c}1462(81) \\
215(12) \\
34(2) \\
43(2) \\
37(2) \\
25(1)\end{array}$ & $\begin{array}{c}665(73) \\
136(15) \\
25(3) \\
26(3) \\
34(4) \\
22(2)\end{array}$ & $<.001$ \\
\hline Mean hospital charges (\$) & 106,405 & 118,931 & $<.001$ \\
\hline 30-d mortality & $60(3)$ & $54(6)$ & .001 \\
\hline 90-d mortality & $99(5)$ & $85(9)$ & $<.001$ \\
\hline 30-d hospital readmission & $257(14)$ & $142(16)$ & .301 \\
\hline 90-d hospital readmission & $415(23)$ & $275(30)$ & $<.001$ \\
\hline \multicolumn{4}{|c|}{ 90-d hospital readmission rate according to readmission diagnosis* } \\
\hline Pulmonary & $213(12)$ & $139(15)$ & .009 \\
\hline Cardiac & $121(7)$ & $80(9)$ & .043 \\
\hline Hematologic & $44(2)$ & $34(4)$ & .051 \\
\hline Renal & $143(8)$ & $105(12)$ & .002 \\
\hline Infectious & $182(10)$ & $136(15)$ & $<.001$ \\
\hline Neurologic & $27(1)$ & $15(2)$ & .741 \\
\hline Gastrointestinal & $15(<1)$ & $<11(<1)$ & .882 \\
\hline Pain & $<11 \dagger$ & $<11 \dagger$ & .135 \\
\hline
\end{tabular}

Values are presented as $\mathrm{n}(\%)$ or median (interquartile range). ${ }^{*}$ More than one category may be present per patient; 287 patients were uncategorized. $\dagger$ Cells representing $\leq 10$ were suppressed per Surveillance, Epidemiology and End Results-Medicare guidelines.

abdominopelvic surgery. ${ }^{7}$ In addition, opioid dose among 450 patients was independently associated with delayed time to healing for chronic wounds, after taking into account pain level. ${ }^{34}$ Lastly, in a large, propensity-matched analysis of the SEER-Medicare database, opioid use after lobectomy for stage I NSCLC was associated with decreased survival and decreased cancer-specific survival. ${ }^{35}$

Opioid using patients have been shown in other studies to demonstrate an increased burden of cardiovascular disease. An analysis of 29,025 patients enrolled in the Reasons for Geographic and Racial Differences in Stroke prospective cohort study revealed that, after adjustment for coronary risk factors, prescription opioid use was associated with an increased risk of cardiovascular death during the study duration. ${ }^{36}$ In addition, chronic analgesia use with opioid or cyclooxygenase-2 therapy has been shown to be associated with a higher risk of myocardial infarction. ${ }^{37,38}$
Separately, without adjusting for opioid use, chronic pain has been shown to be associated with an increased risk of cardiovascular disease. ${ }^{39}$ Plausible mechanisms may include decreased vascular endothelial growth factor expression among cultured myocytes subject to hypoxia, ${ }^{40,41}$ increased arterial stiffness, ${ }^{42}$ or other unidentified pathways.

Opioid use-reduction strategies are actively being developed, largely in the postoperative setting. The implementation of an enhanced recovery after thoracic surgery pathway, which included the use of multimodal analgesia, was associated with a significant reduction in opioid use after surgery. ${ }^{43}$ Other interventions have also been successful, including an intervention bundle consisting of patient and provider education that included the use of multimodal analgesia and resulted in a significant reduction in prescribed opioid drugs without an increase in postoperative pain. ${ }^{44}$ A large quality improvement study involving a regional health system over a 16-month period with more than a million clinical encounters also resulted in a rather substantial $58 \%$ reduction in prescribed opioid medications. $^{45}$

There are several limitations to this retrospective study. Although we performed a detailed propensity scorematching analysis, there is a risk of confounding with variables that were not measured with Medicare claims files. These may include preoperative functional status, physiologic reserve, detailed psychiatric illness, and comorbid conditions predisposing to chronic pain that are not included in the Charlson comorbidity score. In addition, our study population represents a population-based cancer registry with those individuals enrolled in Medicare and older than age 65 years, which may represent different kinds of patients than those sampled in other database studies. Strengths of this study include the use of detailed and comprehensive prescription records, SEER cancer registry data, and large population-level analysis.

\section{CONCLUSIONS}

We identified that chronic opioid users represent high-risk patients, with nearly doubled 30-day and 90-day mortality as well as increased 90-day hospital readmissions. Readmissions relating to infectious, renal, pulmonary, and cardiac causes were significantly higher among chronic users of opioid drugs. Patients undergoing elective lobectomy for lung cancer should be screened to identify chronic opioid use, and these patients should be optimized medically preoperatively. Consideration should be given toward preoperative opioid use-reduction strategies. Because of the higher rate of readmissions in this patient cohort, careful discharge planning and vigilant postoperative follow-up should be undertaken. 
TABLE 5. Factors associated with 90-day readmission in matched cohort

\begin{tabular}{|c|c|c|c|c|}
\hline Factor & $\frac{\text { Univariable }}{\text { Odds ratio }(95 \% \text { Confidence interval })}$ & $P$ value & $\frac{\text { Multivariable }}{\text { Odds ratio }(95 \% \text { Confidence interval })}$ & $P$ value \\
\hline Opioid use & & $<.001$ & & $<.001$ \\
\hline Naïve & Reference & & Reference & \\
\hline Intermittent & $1.40(1.12-1.74)$ & & $1.35(1.07-1.71)$ & \\
\hline Chronic & $1.67(1.36-2.04)$ & & $1.72(1.40-2.12)$ & \\
\hline Age group (y) & & .088 & & .098 \\
\hline$>80$ & Reference & & Reference & \\
\hline $66-70$ & $0.76(0.56-1.04)$ & & $0.75(0.54-1.04)$ & \\
\hline $71-75$ & $0.73(0.53-1.01)$ & & $0.72(0.51-1.00)$ & \\
\hline $76-80$ & $0.92(0.66-1.29)$ & & $0.91(0.64-1.30)$ & \\
\hline Sex & & $<.001$ & & $<.001$ \\
\hline Male & Reference & & Reference & \\
\hline Female & $0.72(0.60-0.86)$ & & $0.71(0.59-0.87)$ & \\
\hline Race & & .982 & & .408 \\
\hline Non-Hispanic white & Reference & & Reference & \\
\hline Hispanic & $1.00(0.69-1.47)$ & & $0.82(0.54-1.25)$ & \\
\hline Non-Hispanic black & $0.96(0.69-1.34)$ & & $0.76(0.53-1.09)$ & \\
\hline Non-Hispanic other & $0.88(0.42-1.86)$ & & $0.83(0.38-1.84)$ & \\
\hline Year of diagnosis & & .256 & & .274 \\
\hline 2008 & Reference & & Reference & \\
\hline 2009 & $0.99(0.73-1.34)$ & & $1.00(0.73-1.37)$ & \\
\hline 2010 & $1.10(0.82-1.48)$ & & $1.10(0.80-1.49)$ & \\
\hline 2011 & $1.21(0.91-1.61)$ & & $1.26(0.93-1.70)$ & \\
\hline 2012 & $0.89(0.66-1.20)$ & & $0.90(0.66-1.23)$ & \\
\hline 2013 & $0.89(0.66-1.20)$ & & $0.93(0.68-1.28)$ & \\
\hline Urban/rural & & .183 & & .059 \\
\hline Big metro & Reference & & Reference & \\
\hline Metro & $1.12(0.92-1.37)$ & & $1.01(0.79-1.29)$ & \\
\hline Urban & $0.83(0.58-1.20)$ & & $0.70(0.46-1.06)$ & \\
\hline Less urban & $0.88(0.66-1.16)$ & & $0.71(0.50-1.02)$ & \\
\hline Rural/unknown & $0.72(0.45-1.14)$ & & $0.59(0.35-0.99)$ & \\
\hline Marriage status & & .161 & & .044 \\
\hline Married & Reference & & Reference & \\
\hline Not married & $1.19(0.99-1.42)$ & & $1.28(1.05-1.56)$ & \\
\hline Unknown & $1.15(0.71-1.86)$ & & $1.23(0.74-2.05)$ & \\
\hline Education & & .038 & & .044 \\
\hline $0-5.66$ & Reference & & Ref & \\
\hline $5.66-10.5$ & $1.33(0.98-1.80)$ & & $1.42(1.02-1.97)$ & \\
\hline $10.5-19.35$ & $1.17(0.88-1.57)$ & & $1.35(0.95-1.92)$ & \\
\hline$\geq 19.35$ & $1.46(1.10-1.93)$ & & $1.68(1.15-2.47)$ & \\
\hline Poverty & & .435 & & .726 \\
\hline $0-5.24$ & Reference & & Reference & \\
\hline $5.24-10.03$ & $0.90(0.67-1.22)$ & & $0.85(0.61-1.19)$ & \\
\hline $10.03-18.19$ & $0.86(0.65-1.13)$ & & $0.84(0.60-1.19)$ & \\
\hline$\geq 18.19$ & $1.01(0.78-1.32)$ & & $0.90(0.62-1.32)$ & \\
\hline Stage & & .068 & & .185 \\
\hline I & Reference & & Reference & \\
\hline 0 & $1.52(0.14-16.9)$ & & $2.32(0.20-26.8)$ & \\
\hline II & $1.22(0.94-1.58)$ & & $1.18(0.90-1.56)$ & \\
\hline III & $0.97(0.76-1.24)$ & & $0.95(0.73-1.23)$ & \\
\hline IV & $1.65(1.10-2.48)$ & & $1.53(1.00-2.34)$ & \\
\hline Unknown & $0.71(0.42-1.20)$ & & $0.72(0.39-1.32)$ & \\
\hline
\end{tabular}


TABLE 5. Continued

\begin{tabular}{|c|c|c|c|c|}
\hline Factor & $\frac{\text { Univariable }}{\text { Odds ratio }(95 \% \text { Confidence interval })}$ & $P$ value & $\frac{\text { Multivariable }}{\text { Odds ratio }(95 \% \text { Confidence interval) }}$ & $P$ value \\
\hline Tumor histology & & .004 & & .416 \\
\hline Adenocarcinoma & Reference & & Reference & \\
\hline Large cell & $1.53(0.97-2.41)$ & & $1.23(0.75-2.00)$ & \\
\hline Other/NOS & $1.08(0.74-1.59)$ & & $1.14(0.74-1.76)$ & \\
\hline Squamous & $1.38(1.15-1.66)$ & & $1.18(0.96-1.45)$ & \\
\hline Tumor size $(\mathrm{cm})$ & & .514 & & .968 \\
\hline$<2$ & Reference & & Reference & \\
\hline $2-3$ & $1.12(0.88-1.42)$ & & $1.03(0.81-1.33)$ & \\
\hline $3-5$ & $1.16(0.92-1.47)$ & & $1.01(0.79-1.30)$ & \\
\hline$\geq 5$ & $1.21(0.92-1.59)$ & & $0.96(0.71-1.31)$ & \\
\hline Surgery approach & & .003 & & .005 \\
\hline Open & Reference & & Reference & \\
\hline Video-assisted thoracoscopic surgery & $0.75(0.62-0.90)$ & & $0.75(0.61-0.92)$ & \\
\hline Neoadjuvant therapy & & .548 & & .851 \\
\hline No & Reference & & Reference & \\
\hline Yes & $1.10(0.80-1.52)$ & & $1.03(0.73-1.46)$ & \\
\hline Grade & & $<.001$ & & .018 \\
\hline Well differentiated & Reference & & Reference & \\
\hline Moderately differentiated & $1.46(1.08-1.98)$ & & $1.31(0.95-1.80)$ & \\
\hline Poorly differentiated & $1.97(1.45-2.66)$ & & $1.64(1.18-2.30)$ & \\
\hline Unknown & $1.64(1.08-2.51)$ & & $1.50(0.95-2.38)$ & \\
\hline Charlson comorbidity score & & $<.001$ & & $<.001$ \\
\hline 0 & Reference & & Reference & \\
\hline 1 & $1.21(0.96-1.53)$ & & $1.16(0.91-1.48)$ & \\
\hline $2+$ & $1.75(1.40-2.17)$ & & $1.58(1.25-2.00)$ & \\
\hline
\end{tabular}

NOS, Not otherwise specified.

\section{Webcast}

You can watch a Webcast of this AATS meeting presentation by going to: https://aats.blob.core.windows.net/media/19\% 20AM/Sunday_May5/203BD/203BD/S62\%20-\%20Doing $\% 20$ the $\%$ 20right $\%$ 20thing $\%$ 20I/S62_6_webcast_050532 890.mp4.

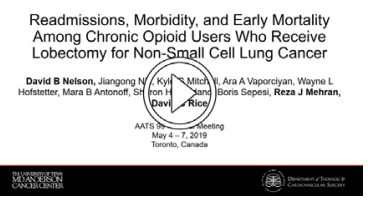

\section{Conflict of Interest Statement}

Authors have nothing to disclose with regard to commercial support.

This study used the linked Surveillance, Epidemiology, and End Results (SEER)-Medicare database, the creation of which reflects efforts of the National Cancer Institute; the Centers for Medicare and Medicaid Services, Office of Research, Development and Information; Information Management Services, Inc; and the SEER Program tumor registries. The interpretation and reporting of these data are the sole responsibility of the authors.

\section{References}

1. Kolodny A, Courtwright DT, Hwang CS, Kreiner P, Eadie JL, Clark TW, et al The prescription opioid and heroin crisis: a public health approach to an epidemic of addiction. Annu Rev Public Health. 2015;36:559-74.

2. Dewan KC, Dewan KS, Idrees JJ, Navale SM, Rosinski BF, Svensson LG, et al Trends and outcomes of cardiovascular surgery in patients with opioid use disorders. JAMA Surg. 2019;154:232-40.

3. Waljee JF, Cron DC, Steiger RM, Zhong L, Englesbe MJ, Brummett CM. Effect of preoperative opioid exposure on healthcare utilization and expenditures following elective abdominal surgery. Ann Surg. 2017;265:715-21.

4. Cron DC, Englesbe MJ, Bolton CJ, Joseph MT, Carrier KL, Moser SE, et al. Preoperative opioid use is independently associated with increased costs and worse outcomes after major abdominal surgery. Ann Surg. 2017;265: 695-701.

5. Al-Hashimi M, Scott SW, Thompson JP, Lambert DG. Opioids and immune modulation: more questions than answers. Br J Anaesth. 2013;111:80-8.

6. Wang J, Barke RA, Charboneau R, Roy S. Morphine impairs host innate immune response and increases susceptibility to Streptococcus pneumoniae lung infection. J Immunol. 2005;174:426-34.

7. Shanmugam VK, Fernandez SJ, Evans KK, McNish S, Banerjee AN, Couch KS, et al. Postoperative wound dehiscence: predictors and associations. Wound Repair Regen. 2015;3:184-90.

8. National Cancer Institute. Surveillance, Epidemiology, and End Results Program; 2018. Available at: https://seer.cancer.gov. Accessed August 31, 2019.

9. Lee JS, Hu HM, Edelman AL, Brummett CM, Englesbe MJ, Waljee JF, et al. New persistent opioid use among patients with cancer after curative-intent surgery. $J$ Clin Oncol. 2017;35:4042-9.

10. Charlson ME, Pompei P, Ales KL, MacKenzie CR. A new method of classifying prognostic comorbidity in longitudinal studies: development and validation. $J$ Chronic Dis. 1987;40:373-83.

11. Klabunde CN, Potosky AL, Legler JM, Warren JL. Development of a comorbidity index using physician claims data. J Clin Epidemiol. 2000;53:1258-67. 
12. Gupta A, Nizamuddin J, Elmofty D, Nizamuddin SL, Tung A, Minhaj M, et al. Opioid abuse or dependence increases 30-day readmission rates after major operating room procedures: a national readmissions database study. Anesthesiology. 2018;128:880-90.

13. Sayal P, Bateman BT, Menendez M, Eikermann M, Ladha KS. Opioid use disorders and the risk of postoperative pulmonary complications. Anesth Analg. 2018;127:767-74.

14. Jain N, Phillips FM, Weaver T, Khan SN. Preoperative chronic opioid therapy: a risk factor for complications, readmission, continued opioid use and increased costs after one- and two-level posterior lumbar fusion. Spine (Phila Pa 1976). 2018;43:1331-8.

15. Barrantes F, Luan FL, Kommareddi M, Alazem K, Yaqub T, Roth RS, et al. A history of chronic opioid usage prior to kidney transplantation may be associated with increased mortality risk. Kidney Int. 2013;84:390-6.

16. Lentine KL, Lam NN, Naik AS, Axelrod DA, Zhang Z, Dharnidharka VR, et al. Prescription opioid use before and after kidney transplant: implications for posttransplant outcomes. Am J Transplant. 2018;18:2987-99.

17. Jain N, Brock JL, Phillips FM, Weaver T, Khan SN. Chronic preoperative opioid use is a risk factor for increased complications, resource use, and costs after cervical fusion. Spine J. 2018;18:1989-98.

18. Bell KL, Shohat N, Goswami K, Tan TL, Kalbian I, Parvizi J. Preoperative opioids increase the risk of periprosthetic joint infection after total joint arthroplasty. J Arthroplasty. 2018;33:3246-32451.e1.

19. Hsiang WR, McGeoch C, Lee S, Cheung W, Becher R, Davis KA, et al. Opioid dependency is independently associated with inferior clinical outcomes after trauma. Injury. 2019;50:192-6.

20. Kim Y, Cortez AR, Wima K, Dhar VK, Athota KP, Schrager JJ, et al. Impact of preoperative opioid use after emergency general surgery. J Gastrointest Surg. 2018;22:1098-103.

21. Hilliard PE, Waljee J, Moser S, Metz L, Mathis M, Goesling J, et al. Prevalence of preoperative opioid use and characteristics associated with opioid use among patients presenting for surgery. JAMA Surg. 2018;153:929-37.

22. Jiang X, Orton M, Feng R, Hossain E, Malhotra NR, Zager EL, et al. Chronic opioid usage in surgical patients in a large academic center. Ann Surg. 2017; 265:722-7.

23. Safaii N, Kazemi B. Effect of opium use on short-term outcome in patients undergoing coronary artery bypass surgery. Gen Thorac Cardiovasc Surg. 2010; 58:62-7.

24. Edelman EJ, Gordon KS, Crothers K, Akgün K, Bryant KJ, Becker WC, et al. Association of prescribed opioids with increased risk of community-acquired pneumonia among patients with and without HIV. JAMA Intern Med. 2019; 179:297-304

25. Wiese AD, Griffin MR, Schaffner W, Stein CM, Greevy RA, Mitchel EF Jr, et al. Opioid analgesic use and risk for invasive pneumococcal diseases: a nested casecontrol study. Ann Intern Med. 2018;168:396-404.

26. Bigliardi PL, Neumann C, Teo YL, Pant A, Bigliardi-Qi M. Activation of the delta-opioid receptor promotes cutaneous wound healing by affecting keratinocyte intercellular adhesion and migration. Br J Pharmacol. 2015;172:501-14.

27. Bigliardi-Qi M, Gaveriaux-Ruff C, Zhou H, Hell C, Bady P, et al. Deletion of $\delta$ opioid receptor in mice alters skin differentiation and delays wound healing. Differentiation. 2006; 74:174-85.

28. Martin JL, Koodie L, Krishnan AG, Charboneau R, Barke RA, Roy S. Chronic morphine administration delays wound healing by inhibiting immune cell recruitment to the wound site. Am J Pathol. 2010;176:786-99.

29. Rook JM, Hasan W, McCarson KE. Morphine-induced early delays in wound closure: involvement of sensory neuropeptides and modification of neurokinin receptor expression. Biochem Pharmacol. 2009;77:1747-55.

30. Rook JM, Hasan W, McCarson KE. Temporal effects of topical morphine application on cutaneous wound healing. Anesthesiology. 2008;109:130-6.

31. Martin JL, Charboneau R, Barke RA, Roy S. Chronic morphine treatment inhibits LPS-induced angiogenesis: implications in wound healing. Cell Immunol. 2010;265:139-45.

32. Lam CF, Chang PJ, Huang YS, Sung YH, Huang CC, Lin MW, et al. Prolonged use of high-dose morphine impairs angiogenesis and mobilization of endothelial progenitor cells in mice. Anesth Analg. 2008;107:686-92.

33. McLaughlin PJ, Immonen JA, Zagon IS. Topical naltrexone accelerates fullthickness wound closure in type 1 diabetic rats by stimulating angiogenesis. Exp Biol Med (Maywood). 2013;238:733-43.

34. Shanmugam VK, Couch KS, McNish S, Amdur RL. Relationship between opioid treatment and rate of healing in chronic wounds. Wound Repair Regen. 2017;25: 120-30.
35. Nelson DB, Cata JP, Niu J, Mitchell KG, Vaporciyan AA, Antonoff MB, et al Persistent opioid use is associated with worse survival after lobectomy for stage I non-small cell lung cancer. Pain. 2019;160:2365-73.

36. Khodneva Y, Muntner P, Kertesz S, Kissela B, Safford M. Prescription opioid use and risk of coronary heart disease, stroke, and cardiovascular death among adults from a prospective cohort (REGARDS) study. Pain Med. 2016;17: 444-55.

37. Carman WJ, Su S, Cook SF, Wurzelmann JI, McAfee A. Coronary heart disease outcomes among chronic opioid and cyclooxygenase-2 users compared with a general population cohort. Pharmacoepidemiol Drug Saf. 2011;20:754-62.

38. Li L, Setoguchi S, Cabral H, Jick S. Opioid use for noncancer pain and risk of myocardial infarction amongst adults. J Intern Med. 2013;273: 511-26.

39. Fayaz A, Watt HC, Langford RM, Donaldson LJ. The association between chronic pain and cardiac disease: a cross-sectional population study. Clin J Pain. 2016;32:1062-8.

40. Balasubramanian S, Ramakrishnan S, Charboneau R, Wang J, Barke RA, Roy S Morphine sulfate inhibits hypoxia-induced vascular endothelial growth factor expression in endothelial cells and cardiac myocytes. J Mol Cell Cardiol. 2001;33:2179-87.

41. Roy S, Balasubramanian S, Wang J, Chandrashekhar Y, Charboneau R, Barke R. Morphine inhibits VEGF expression in myocardial ischemia. Surgery. 2003;134 336-44.

42. Reece AS, Hulse GK. Impact of lifetime opioid exposure on arterial stiffness and vascular age: cross-sectional and longitudinal studies in men and women. BMJ Open. 2014;4:e004521.

43. Martin LW, Sarosiek BM, Harrison MA, Hedrick T, Isbell JM, Krupnick AS, et al. Implementing a thoracic enhanced recovery program: lessons learned in the first year. Ann Thorac Surg. 2018;105:1597-604.

44. Hartford LB, Van Koughnett JAM, Murphy PB, Vogt KN, Hilsden RJ, Clarke CF, et al. Standardization of outpatient procedure (STOP) narcotics: a prospective non-inferiority study to reduce opioid use in outpatient general surgical procedures. J Am Coll Surg. 2019;228:81-8.e1.

45. Meisenberg BR, Grover J, Campbell C, Korpon D. Assessment of opioid prescribing practices before and after implementation of a health system intervention to reduce opioid overprescribing. JAMA Netw Open. 2018;1: e182908.

Key Words: lung cancer, lobectomy, opioid dependency, perioperative morbidity

\section{Discussion}

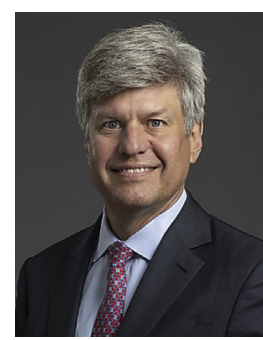

Dr Michael J. Liptay (Chicago, Ill). I would like to congratulate Dr Nelson and his colleagues for a well-presented and timely study. Thanks also for providing the manuscript well beforehand.

The opioid crisis is front and center and recognized as a major challenge facing our health care system. Surgeons are often unwitting contributors-a presentation just yesterday concluded that 1 out of 14 patients undergoing major cardiothoracic surgeries become chronic opioid users. You have shown with your data today the serious potential risk for increased complications and mortality if a future lobectomy is needed.

It was interesting to me that more than half of patients in all the groups underwent a thoracotomy and not a minimally invasive approach. The chronic opioid users who seemingly would benefits most from a minimally invasive approach underwent a video-assisted thoracoscopic 
surgical approach only $35 \%$ of the time compared with $44 \%$ of the opioid naïve cohort. I know this isn't your institution-it's a database review — but do you have any thoughts on that?

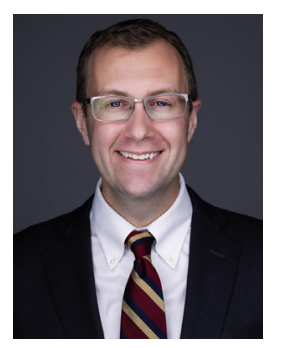

Dr David B. Nelson (Houston, Tex). That is a great question. A benefit of looking at Surveillance, Epidemiology and End Results-Medicare data is you look at what happens nationwide as opposed to at an experienced tertiary care center.

Dr Liptay. I don't know what the lag is in entering data, but you looked at data from 2008 to 2013. Do you think if you looked at data from 2013 to 2018 it would be different?

Dr Nelson. That's certainly a possibility. Others have published data showing that the use of minimally invasive approaches in general, including robotic surgery, is still increasing, with fewer open cases being performed. There is an update in the Surveillance, Epidemiology and End Results-Medicare file to 2015, which may help clarify this. Certainly, the use of VATS might be higher more recently.

Dr Liptay. You noted that using video-assisted thoracoscopic surgical approach translated into a decreased risk of 90-day readmissions of a hazard ratio of 0.75 , I think.

You say in your Conclusions that you would need to optimize medically these chronic opioid users before taking them to surgery, but you also mention that chronic opioid users are often of poor economic status, less educated, continue to smoke, and are less compliant with recommendations. How do you medically optimize these people in your practice?

Dr Nelson. It may be difficult to tease out the differences of the effect of opioids use on readmission opposed to other factors that could be contributing, such as smoking or medication compliance. It may be the case that, even without knowing opioid use status, a provider would know whether or not a patient needs medical optimization. Obviously, if you are still smoking and other factors you mentioned, those are risk factors that need to be targeted. I think everything you can do to optimize a patient before surgery is important. Among these factors-based on our research-may be opioid reduction.

Dr Liptay. Thanks very much.

Dr Nelson. Thank you.

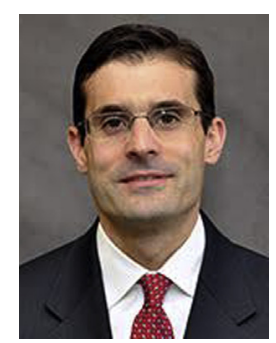

Dr Felix Fernandez (Atlanta, Ga). Very well presented and certainly a very timely topic. Did you also query postoperative opioid use in the different patient cohorts in the Surveillance, Epidemiology and End ResultsMedicare dataset to look at how that may have influenced or been associated with the outcomes as well?

Dr Nelson. We haven't looked at that for morbidity. That's a great question. 
APPENDIX E1. COMMON PROCEDURAL TERMINOLOGY (CPT)/INTERNATIONAL CLASSIFICATION OF DISEASES, 9TH EDITION (ICD-9)/INTERNATIONAL CLASSIFICATION OF DISEASES FOR ONCOLOGY, THIRD EDITION (ICD-O-3)/NATIONAL DRUG CODE (NDC) CODE IDENTIFICATION

1. Non-small cell lung cancer

Histology code 8010-8015, 8020-8022, 8030-8040, $8046,8050-8052,8070-8084,8090-8110,8120-$ $8131,8140-8156,8160-8162,8170-8175,8180$, $8190-8221,8230-8231,8240-8263,8270-8280$, $8290-8337,8340-8347,8350-8390,8400-8562$, 8570-8576, 8580-8671, 8940-8941

2. Surgery codes

a. Pneumonectomy

i. VATS: $32.5,32.50$; CPT: 32671

ii. Open: 32.59 ; CPT: $32440,32445,32488$

b. Sleeve lobectomy

i. Open: CPT: 32486

ii. No ICD-9 code

iii. No VATS equivalent

c. Sleeve pneumonectomy

i. Open: CPT: 32442

ii. No ICD-9 code

iii. No VATS equivalent

d. Lobar resection

i. VATS: $32.4,32.41$; CPT: 32663

ii. Open: 32.49 , CPT: 32480

e. Sublobar resection i. VATS wedge: 32.20 ; CPT: $32602,32607,32608$, 32657, 32666, 32667, 32668

ii. Open wedge: 32.29; CPT: 32095, 32096, 32097; 32505, 32506, 32507

iii. VATS segmentectomy: 32.3, 32.30; CPT: 32669

iv. Open segmentectomy: 32.39; CPT: 32484

3. Opioid (by generic name)

f. Flag

i. Oxycodone

ii. Tramadol

iii. Codeine

iv. Dihydrocodeine

v. Fentanyl

vi. Oxymorphone

vii. Morphine

viii. Methadone

ix. Meperidine

x. Hydromorphone

xi. Hydrocodone

xii. Tapentadol

xiii. Pentazocine

xiv. Butorphanol

xv. Nalbuphine

xvi. Pentazocine

xvii. Buprenorphine

xviii. Propoxyphene

xix. Levorphanol

g. Without the following flags

i. Butalbital

ii. Guaifenesin

iii. Homatropine

iv. Phenylephrine

v. Pseudoephedrine 

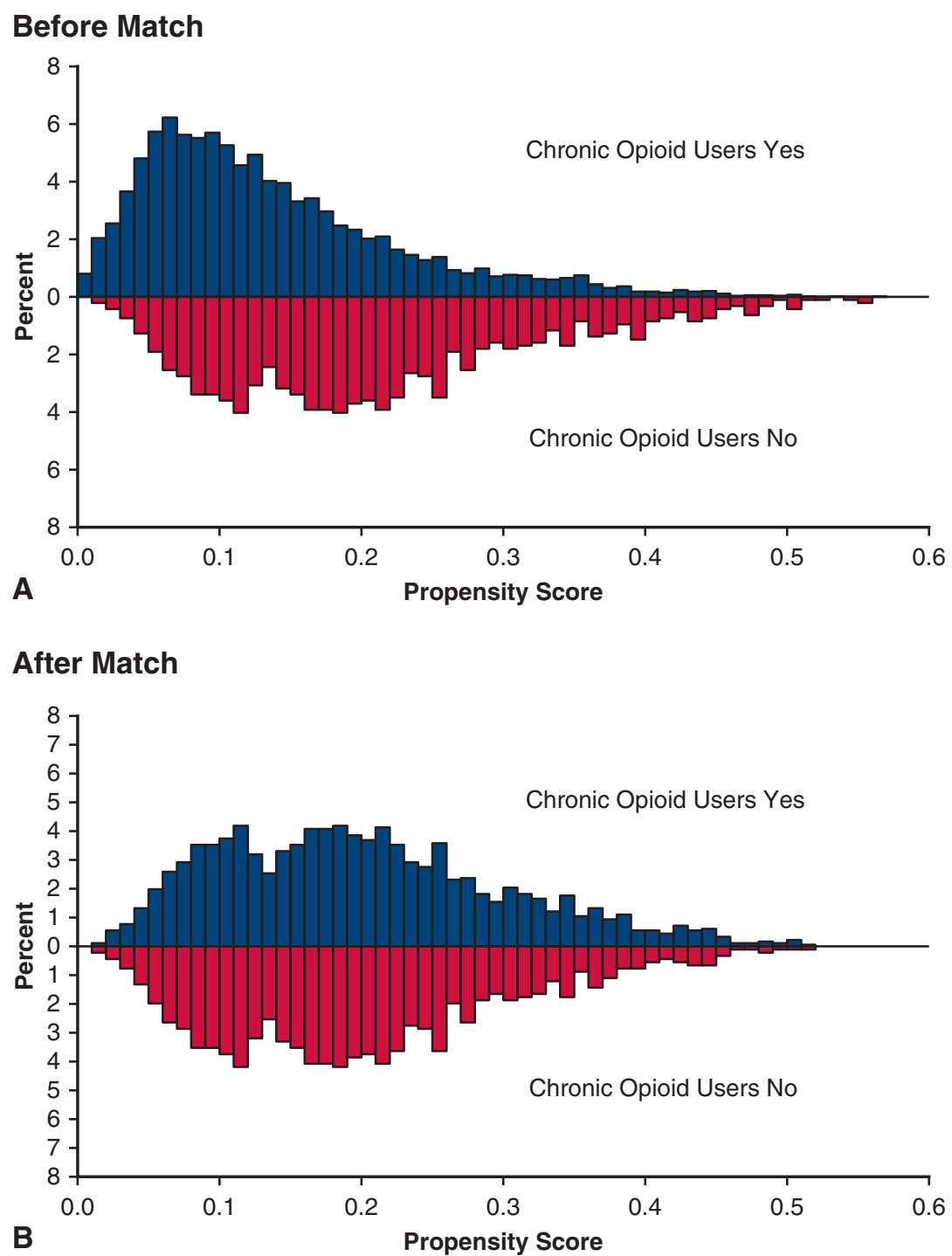

FIGURE E1. Mirror histograms indicating propensity score distribution before and after propensity matching.

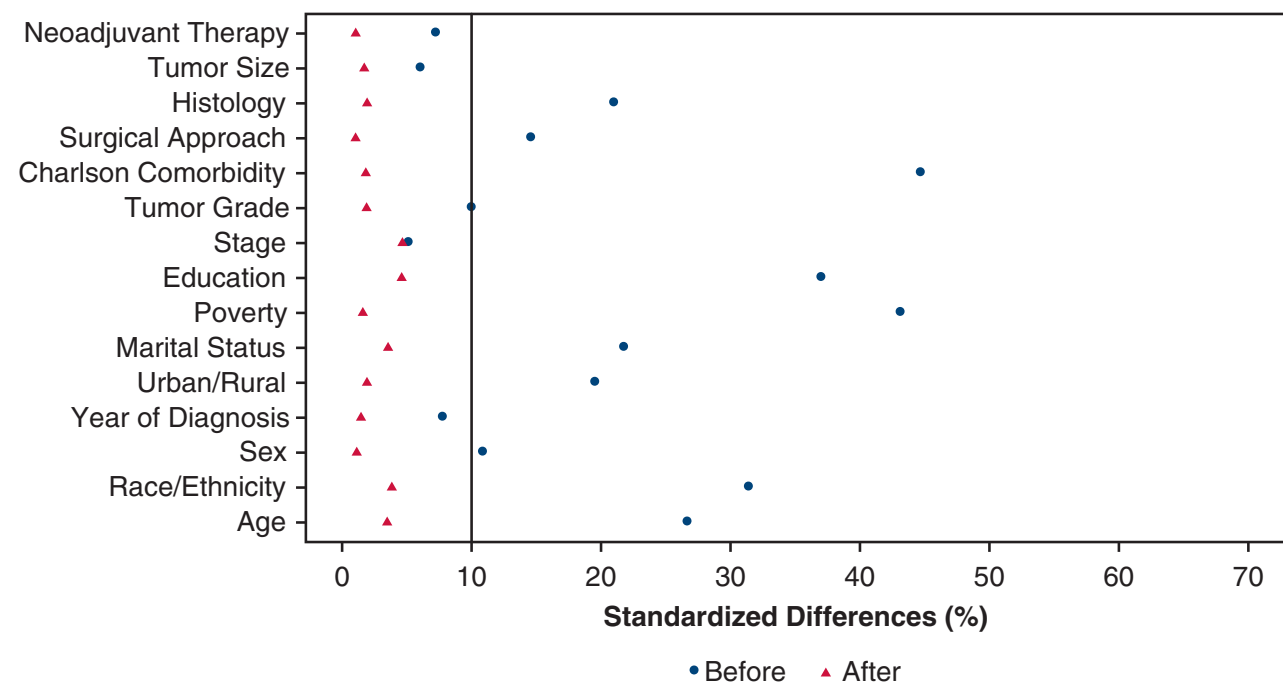

FIGURE E2. Standardized mean differences before and after propensity matching. 
TABLE E1. International Classification of Diseases-Ninth Revision (ICD-9) diagnosis codes upon hospital readmission

\begin{tabular}{|c|c|c|}
\hline Complication category & Diagnosis & ICD-9 diagnosis code \\
\hline \multirow[t]{8}{*}{ Respiratory } & Chronic obstructive pulmonary disease & $491.21491 .22492 *$ \\
\hline & Aspiration & 507.* 508.8* 508.9* \\
\hline & Other & $997.3^{*}$ \\
\hline & Pleurisy & $511 *$ \\
\hline & Pneumothorax & $512 *$ \\
\hline & Pulmonary insufficiency & $518 *$ \\
\hline & Respiratory arrest & $799.1^{*}$ \\
\hline & Respiratory distress & 786.* except $786.5^{*}$ \\
\hline \multirow[t]{7}{*}{ Cardiac } & Myocardial infarction & $410 * 411 *$ \\
\hline & Dysrhythmias & $427.0 * 427.1 * 427.2 * 427.3 * 427.4 * 427.89427 .9 *$ \\
\hline & Heart failure & $\begin{array}{l}428.0428 .1428 .2428 .21428 .23428 .3428 .31428 .33 \\
\quad 428.4428 .41428 .43428 .9\end{array}$ \\
\hline & Other & 997.1785 .51998 .01 \\
\hline & Pericarditis & $420 * *$ \\
\hline & Cardiac arrest & 427.5 \\
\hline & Chest pain NOS & 413.* \\
\hline \multirow[t]{4}{*}{ Heme } & Blood loss anemia & $285.1^{*}$ \\
\hline & $\begin{array}{l}\text { Thromboembolic events (deep vein thrombosis and } \\
\text { pulmonary embolism) }\end{array}$ & $415.1 * 453.4 *$ \\
\hline & Embolism & $444 . *$ \\
\hline & Gastrointestinal bleed & $578 * *$ \\
\hline Gastrointestinal & Obstruction, nausea, or vomiting & $560 .^{*}, 536 *, 787.0^{*}$ \\
\hline \multirow[t]{3}{*}{ Renal } & Acute kidney injury & 584.* 586.* \\
\hline & Electrolyte/fluid & 276.* \\
\hline & Syncope & 780.2 \\
\hline \multirow[t]{8}{*}{ Infectious } & Pneumonia & $481 * * 482 . * 483 . * 507 . * 486 . * 513 . *$ \\
\hline & Wound infection & $998.5^{*}, 998.02$ \\
\hline & Cellulitis & $682 . *$ \\
\hline & Bacteremia, septicemia or SIRS & 038.* $790.7995 .9 *$ \\
\hline & Urinary tract infection & 599.0 \\
\hline & Shock & $785.5^{*}$ \\
\hline & Empyema & $510 .^{*}$ \\
\hline & Wound & 875.*, 998.* \\
\hline \multirow[t]{3}{*}{ Neuro } & Cerebrovascular attack/transient ischemic attack & 434.* 435.*, 431 \\
\hline & Postoperative stroke or hemorrhage & 997.02 \\
\hline & Encephalopathy & $348 .^{*}$ \\
\hline Pain & Pain & $\begin{array}{l}292.0 \\
292.11,292.12,292.2,292.8,292.82,292.83,292.84, \\
\quad 292.85,292.89,292.9 \\
338.12,338.18,338.19 \\
786.5^{*} \\
965.00,965.01,965.02,965.09 \\
967.0,967.6,967.8-968.0,969 .^{*}, 970.1,970.8,970.81, \\
\quad 970.89,970.9,975.2,975.4,975.6,976.1\end{array}$ \\
\hline
\end{tabular}

NOS, Not otherwise specified; SIRS, systemic inflammatory response syndrome. 
TABLE E2. Matched patient, tumor, and treatment characteristics after propensity matching 1:2 according to chronic opioid use

\begin{tabular}{|c|c|c|c|}
\hline Characteristic & $\begin{array}{c}\text { Preoperative naïve or } \\
\text { intermittent opioid } \\
\text { use }(n=1816)\end{array}$ & $\begin{array}{l}\text { Preoperative chronic } \\
\text { opioid use }(n=908)\end{array}$ & $P$ value \\
\hline Age $(y)$ & & & .865 \\
\hline $66-70$ & $738(41)$ & $355(39)$ & \\
\hline $71-75$ & $573(32)$ & $290(32)$ & \\
\hline $76-80$ & 349 (19) & $184(20)$ & \\
\hline$>80$ & $156(9)$ & 79 (34) & \\
\hline Sex & & & .781 \\
\hline Male & 712 (39) & $351(39)$ & \\
\hline Female & $1104(61)$ & $557(61)$ & \\
\hline Year of diagnosis & & & .999 \\
\hline 2008 & 317 (17) & 157 (17) & \\
\hline 2009 & $281(15)$ & $140(15)$ & \\
\hline 2010 & $287(16)$ & $147(16)$ & \\
\hline 2011 & $322(18)$ & $159(18)$ & \\
\hline 2012 & 311 (17) & $158(17)$ & \\
\hline 2013 & $298(16)$ & $147(16)$ & \\
\hline Race/ethnicity & & & .823 \\
\hline Non-Hispanic white & $1563(86)$ & $770(85)$ & \\
\hline Non-Hispanic black & $130(7)$ & $73(8)$ & \\
\hline Hispanic & $97(5)$ & $52(6)$ & \\
\hline Non-Hispanic other & $26(1)$ & $13(2)$ & \\
\hline Urban/rural & & & .994 \\
\hline Big metro & $860(47)$ & $435(48)$ & \\
\hline Less urban & $226(12)$ & $112(12)$ & \\
\hline Metro & $525(29)$ & 259 (29) & \\
\hline Rural/unknown & $83(5)$ & $39(4)$ & \\
\hline Urban & $122(7)$ & $63(7)$ & \\
\hline Marital status & & & .690 \\
\hline Married & 808 (44) & 402 (44) & \\
\hline Not married & $943(52)$ & $479(53)$ & \\
\hline Unknown & $65(4)$ & $27(3)$ & \\
\hline$\%$ Below poverty line within ZIP code & & & .985 \\
\hline $0-5.4$ & $268(15)$ & $136(15)$ & \\
\hline $5.4-10.3$ & $552(30)$ & $270(30)$ & \\
\hline $10.3-18.8$ & $345(19)$ & $176(19)$ & \\
\hline$\geq 18.8$ & $651(36)$ & $326(33)$ & \\
\hline$\%$ With no high school education within ZIP code & & & .731 \\
\hline $0-5.8$ & $254(14)$ & $140(15)$ & \\
\hline $5.8-11.0$ & $545(30)$ & $274(30)$ & \\
\hline $11.0-20.0$ & $410(23)$ & $194(21)$ & \\
\hline$\geq 20.0$ & $607(33)$ & $300(33)$ & \\
\hline Charlson comorbidity score & & & .904 \\
\hline 0 & $517(28)$ & $252(28)$ & \\
\hline 1 & $589(32)$ & $294(33)$ & \\
\hline $2+$ & $710(39)$ & $362(40)$ & \\
\hline Histology & & & .973 \\
\hline Adenocarcinoma & $1017(56)$ & $512(56)$ & \\
\hline Squamous & $631(35)$ & $314(35)$ & \\
\hline Large cell & $56(3)$ & $32(4)$ & \\
\hline Other/NOS & $107(6)$ & $50(6)$ & \\
\hline
\end{tabular}


TABLE E2. Continued

\begin{tabular}{|c|c|c|c|}
\hline Characteristic & $\begin{array}{c}\text { Preoperative naïve or } \\
\text { intermittent opioid } \\
\text { use }(n=1816)\end{array}$ & $\begin{array}{l}\text { Preoperative chronic } \\
\text { opioid use }(n=908)\end{array}$ & $P$ value \\
\hline Grade & & & .975 \\
\hline Well differentiated & $235(13)$ & $122(13)$ & \\
\hline Moderately differentiated & $786(43)$ & $387(43)$ & \\
\hline Poorly differentiated & $666(37)$ & $336(37)$ & \\
\hline Unknown & $129(7)$ & $63(7)$ & \\
\hline Stage & & & .935 \\
\hline I & $1147(63)$ & $582(34)$ & \\
\hline II & $224(12)$ & 119 (13) & \\
\hline III & $303(17)$ & $140(15)$ & \\
\hline IV & $74(4)$ & $37(4)$ & \\
\hline Unknown/0 & $68(4)$ & $30(3)$ & \\
\hline Tumor size $(\mathrm{cm})$ & & & .981 \\
\hline$<2$ & $478(26)$ & $233(26)$ & \\
\hline $2-3$ & $504(28)$ & $254(28)$ & \\
\hline $3-5$ & $548(30)$ & $279(31)$ & \\
\hline$\geq 5$ & $286(67)$ & $142(16)$ & \\
\hline Approach & & & .799 \\
\hline Open & $1177(65)$ & $584(64)$ & \\
\hline Video-assisted thoracoscopic surgery & $639(35)$ & $324(36)$ & \\
\hline Chemotherapy & & & .737 \\
\hline None & $1262(69)$ & $636(70)$ & \\
\hline Neoadjuvant & $118(7)$ & $64(7)$ & \\
\hline Adjuvant & $436(24)$ & $208(23)$ & \\
\hline Radiation & & & .242 \\
\hline None & $1510(83)$ & 777 (86) & \\
\hline Neoadjuvant & $57(3)$ & $22(2)$ & \\
\hline Adjuvant & 249 (14) & 109 (12) & \\
\hline
\end{tabular}

Values are presented as n (\%). NOS, Not otherwise specified. 